\title{
Lepton Number Violation in Higgs Decay at LHC
}

\author{
Alessio Maiezza, ${ }^{1, *}$ Miha Nemevšek,${ }^{2, \dagger}$ and Fabrizio Nesti ${ }^{3}, \ddagger$ \\ ${ }^{1}$ IFIC, Universitat de València-CSIC, Apt. Correus 22085, E-46071 València, Spain \\ ${ }^{2}$ Jožef Stefan Institute, Ljubljana, Slovenia \\ ${ }^{3}$ Ruđer Bošković Institute, Bijenička cesta 54, 10000, Zagreb, Croatia
}

(Dated: October 8, 2018)

\begin{abstract}
We show that within the Left-Right symmetric model, lepton number violating decays of the Higgs boson can be discovered at the LHC. The process is due to the mixing of the Higgs with the triplet that breaks parity. As a result, the Higgs can act as a gateway to the origin of heavy Majorana neutrino mass. To assess the LHC reach, a detailed collider study of the same-sign di-leptons plus jets channel is provided. This process is complementary to the existing nuclear and collider searches for lepton number violation and can probe the scale of parity restoration even beyond other direct searches.
\end{abstract}

PACS numbers: 14.80.Bn, 11.30.-j, 12.60.-i, 13.35.Hb

The discovery of the Higgs boson [1,2] allows to test the mechanism of elementary particle mass generation at the LHC [3]. Compared to this success, the problem of neutrino mass in the Standard Model (SM) appears acute. Neutrinos may be their own antiparticles [4], and lead to lepton number violation (LNV). The canonical way of searching for LNV, neutrino-less double beta decay $(0 \nu 2 \beta)$ [5], can be induced by light Majorana neutrinos or by new physics [6]. The latter, needed for neutrino mass, can be provided by the celebrated seesaw mechanism [7-11]. In particular, Left-Right symmetric models (LRSM) [12], designed to explain parity violation of weak interactions [13], embed naturally the seesaw [7, 8]. With the left-right (LR) scale in the $\mathrm{TeV}$ range, $0 \nu 2 \beta$ may be dominated by heavy Majorana neutrino $(N)$ exchange [14, 15], which may become favored in view of the cosmological bound on light neutrino masses.

A direct strategy for LNV searches at hadron colliders was suggested in [16] by Keung and Senjanović (KS) [17]. The KS production of heavy Majorana neutrinos would reveal LNV and relate directly to $0 \nu 2 \beta[15,18]$ and lepton flavor violation (LFV) $[19,20]$. The Dirac mass is predicted [21] and may be tested at the LHC through LNV decays, uncovering the underlying seesaw mechanism and connecting to electric dipole moments [21, 22]. Indirect constraints [23-27] played an important role and comprehensive analyses [28, 29] allow the LR scale well within the $\sim 6 \mathrm{TeV}$ reach of the LHC [30].

In this Letter we show that LHC can probe a new channel, connecting Higgs physics to restoration of parity. We exploit the fact that the SM Higgs can have a sizeable mixing with the triplet that breaks spontaneously LR symmetry and provides a mass to $N$. Through this mixing the Higgs can decay to a pair of $N$ [31]. Therefore, it can probe their Yukawa couplings via a LNV final state with two same or opposite sign charged leptons and four jets, as shown on Fig. 1. The relevant range of $N$ masses

\footnotetext{
*amaiezza@ific.uv.es

$\dagger$ miha.nemevsek@ijs.si

‡ fabrizio.nesti@irb.hr
}

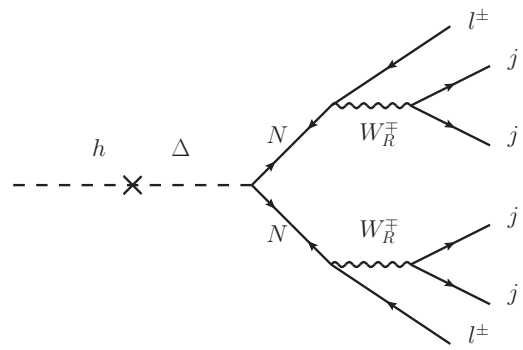

FIG. 1. Dominant diagram leading to LNV Higgs decay.

typically leads to displaced vertices. Higgs decay to $\mathrm{RH}$ neutrinos was mentioned in [32] and studied in [33] with effective operators, pointing out the LNV character and vertex displacement. Here, in the LRSM, the LNV decay is probing the origin of $N$ masses, just as the standard decays test the origin of charged fermion masses. The Higgs can thus act as a portal to LNV, complementary to $0 \nu 2 \beta$ and the KS reaction.

To estimate the LHC sensitivity, we implement the model [34], perform a simulation of the signal and the expected SM background, and devise cuts. To further enhance the search, we simulate the displaced vertices from $N$ decay and highlight their importance. Given the current limits on the Higgs mixing [35] (see outlook [36]), a discovery is possible for a high LR scale, beyond the reach of other direct searches.

We conclude with a discussion on alternative models with potential LNV Higgs decays and a short outlook on the related search at $e^{+} e^{-}$colliders.

Left-Right symmetry and Higgs mixing. LeftRight symmetric models [12], based on the gauge group $S U(2)_{L} \times S U(2)_{R} \times U(1)_{B-L}$, contain a right-handed $(\mathrm{RH})$ gauge boson $W_{R}$ and three $\mathrm{RH}$ Majorana neutrinos. The scalar sector of the minimal LRSM $[7,8]$ features a complex bi-doublet $\Phi \in\left(2_{L}, 2_{R}, 0\right)$ and a pair of triplets $\Delta_{L} \in\left(3_{L}, 1_{R}, 2\right), \Delta_{R} \in\left(1_{L}, 3_{R}, 2\right)$ :

$$
\Phi=\left(\begin{array}{cc}
\phi_{1}^{0} & \phi_{2}^{+} \\
\phi_{1}^{-} & \phi_{2}^{0}
\end{array}\right), \Delta_{L, R}=\left(\begin{array}{cc}
\delta^{+} / \sqrt{2} & \delta^{++} \\
\delta^{0} & -\delta^{+} / \sqrt{2}
\end{array}\right)_{L, R} .
$$

In the minimal LRSM, LR symmetry is restored at 
high energies. The potential exhibits spontaneous breaking and since the original work [13] it has been the subject of several studies [25, 31, 37-39]. We focus on the mixing between the triplet and the SM-like Higgs and display the relevant terms:

$$
\begin{aligned}
\mathcal{V}= & -\mu_{1}^{2}\left(\Phi^{\dagger} \Phi\right)-\mu_{2}^{2}\left(\widetilde{\Phi} \Phi^{\dagger}+\widetilde{\Phi}^{\dagger} \Phi\right)-\mu_{3}^{2}\left(\Delta_{R}^{\dagger} \Delta_{R}\right) \\
& +\lambda\left(\Phi^{\dagger} \Phi\right)^{2}+\rho\left(\Delta_{R}^{\dagger} \Delta_{R}\right)^{2}+\alpha\left(\Phi^{\dagger} \Phi\right)\left(\Delta_{R}^{\dagger} \Delta_{R}\right)
\end{aligned}
$$

The trace on the parenthesis is implied and $\widetilde{\Phi} \equiv \sigma_{2} \Phi^{*} \sigma_{2}$. The results below hold for restoration of both generalized parity $\mathcal{P}$ and charge-conjugation $\mathcal{C}$ [26].

The parameters $\mu$ are fixed in the usual way, $\mu_{1}^{2}=$ $2 \lambda v^{2}+\alpha v_{R}^{2}, \mu_{2}^{2}=0, \mu_{3}^{2}=\alpha v^{2}+2 \rho v_{R}^{2}$, and neutral scalars develop VEVs. The LR-breaking scale is set by $\left\langle\delta_{R}^{0}\right\rangle=v_{R}$ and electroweak symmetry breaking is completed by $\left\langle\phi_{1}^{0}\right\rangle=v$. For clarity we stick to the case where $\phi_{2}^{0}$ does not acquire VEV and we suppress higher $v / v_{R}$ terms. In what follows the neutral scalars $\phi$ and $\delta$ are the fluctuations of $\Re\left(\phi_{1}^{0}\right)$ and $\Re\left(\delta_{R}^{0}\right)$.

Expansion of the potential (2) around the minimum gives the following mass matrix for $\phi$ and $\delta$

$$
M^{2}=2\left(\begin{array}{cc}
2 \lambda v^{2} & \alpha v v_{R} \\
\alpha v v_{R} & 2 \rho v_{R}^{2}
\end{array}\right)
$$

Its diagonalization leads to the masses of the physical particles $m_{h}^{2} \simeq 4 \lambda v^{2}-\alpha^{2} v^{2} / \rho, m_{\Delta}^{2} \simeq 4 \rho v_{R}^{2}$. Here $h=$ $\phi \cos \theta-\delta \sin \theta$ is identified with the SM Higgs boson and $\Delta=\delta \cos \theta+\phi \sin \theta$ with the further neutral state. Their mixing angle can be large, $\theta \simeq(\alpha / 2 \rho)\left(v / v_{R}\right)$.

Since $\delta$ is a SM singlet, mixing universally reduces the SM-like Higgs couplings. Recent studies [35] allow for $\sin \theta<0.44$ at $2 \sigma \mathrm{CL}$, nearly independently of the singlet mass.

Heavy Neutrino from Higgs decay. After spontaneous breaking, the Yukawa term $\mathcal{L}_{\Delta}=Y_{N} L_{R}^{T} \Delta_{R} L_{R}+$ h.c., which couples the RH leptonic doublet $L_{R}$ to the triplet Higgs, generates the heavy neutrino mass matrix. This is directly proportional to the LR scale

$$
M_{N}=2 Y_{N} v_{R}, \quad M_{W_{R}}=g v_{R},
$$

where $g=g_{L, R}$ is the $S U(2)_{L, R}$ gauge coupling constant.

To probe the spontaneous origin of $N$ mass, one should observe $\Delta \rightarrow N N$ decays and establish that $\Gamma_{\Delta \rightarrow N N} \propto$ $m_{N}^{2}$. While the production of $\Delta$ is small due to the large LR scale, in the presence of $\theta$ the gluon fusion production appears. Still, $\Delta$ may be heavy enough to be elusive, in fact from perturbativity of $\alpha, \lambda \lesssim 1$, one finds $m_{\Delta} \lesssim$ $5 \mathrm{TeV}(0.1 / \sin \theta)$. More importantly, the SM Higgs can decay to $N N$. Thus the origin of neutrino masses may be probed by the SM Higgs boson.

It is useful to normalize this decay rate to the leading SM channel $h \rightarrow b \bar{b}$

$$
\frac{\Gamma_{N N}}{\Gamma_{b \bar{b}}} \simeq \frac{\tan \theta^{2}}{3}\left(\frac{m_{N}}{m_{b}}\right)^{2}\left(\frac{M_{W}}{M_{W_{R}}}\right)^{2}\left(1-\frac{4 m_{N}^{2}}{m_{h}^{2}}\right)^{\frac{3}{2}}
$$

neglecting the $b \bar{b}$ phase space. Including the QCD running and NLO corrections [40], the ratio is enhanced by a factor $\approx 2$ and is shown on Fig. 2 .

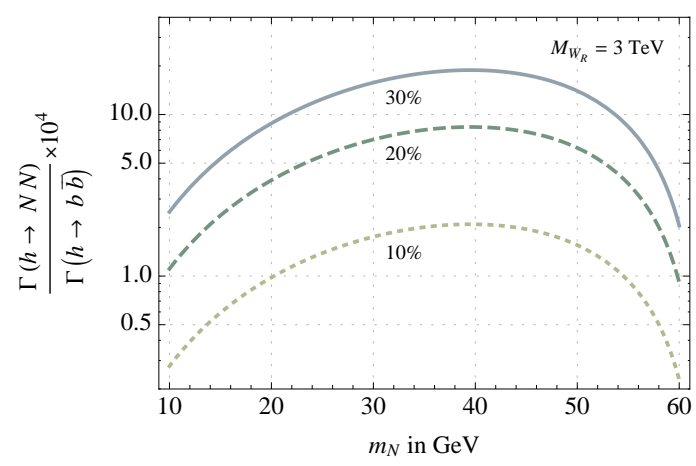

FIG. 2. Decay rate of the SM Higgs $h \rightarrow N N$, normalized to the leading $h \rightarrow b \bar{b}$ channel.

The number of $N$ pairs produced at the $13 \mathrm{TeV}$ LHC run with $100 \mathrm{fb}^{-1}$ luminosity is simple to estimate. Taking the SM Higgs gluon fusion cross-section [41] $\sigma(g g \rightarrow$ $h)=43.9 \mathrm{pb}$ and $\operatorname{Br}(b \bar{b})=57 \%$, one gets $500(2000) h \rightarrow$ $N N$ events for $m_{N}=40 \mathrm{GeV}$ and $\theta=10 \%$ (20\%). This is sufficient motivation for an in-depth collider study.

Lepton number violating Higgs decay at the LHC. After pair-production from Higgs decay, each $N$ decays to a charged lepton and two jets via $W_{R}$, with a $\mathrm{RH}$ charged current quark flavour structure essentially identical to the left-handed one [42]. Due to the Majorana nature of $N$, $50 \%$ of events will result in a final state of two same-sign leptons and four jets with no missing energy, explicitly signalling LNV.

To assess the LHC sensitivity, we extend [34] the FeynRules [43] implementation of the LRSM [44] to include the mixing together with Higgs gluon fusion production. Parton level events are simulated with MadGraph 5 [45], hadronized with Pythia 6 [46] and passed to Delphes 3 [47] for detector simulation. We use MadAnalysis 5 [48] for cuts and event counting. Dedicated software extensions are implemented in each module to study the displaced vertices.

The channel $h \rightarrow \ell^{ \pm} \ell^{ \pm} 4 j$ carries plenty of physical information at parton level. The total invariant mass reconstructs the Higgs mass, while the $\ell j j$ invariant mass reconstructs the $N$ peak. Tagging the lepton flavor identifies the RH analog of the PMNS mixing matrix and the related Majorana mass matrix, as with the KS process $[20,49]$. Notice that with such low $N$ masses, LFV constraints are easily satisfied and one may expect LFV in Higgs decays.

Reconstruction at detector level is more delicate. The Higgs is produced with a boost $\gamma(h) \sim 3$ at $\sqrt{s}=13 \mathrm{TeV}$ and the $N$ is further boosted if $m_{N} \ll m_{h} / 2$. As a result the two jets from $N$ tend to merge. In addition, the jets get closer to the charged lepton and typical lepton isolation cuts may prevent its recognition. Furthermore, the distribution of transverse momentum of the lepton (and the jets) peaks at a fairly low value of $m_{h} / 6 \sim$ $20 \mathrm{GeV}$. Typical detector simulation parameters forbid tight leptons with $p_{T}<10 \mathrm{GeV}$, causing a loss of the 
signal by a factor of 2 . Still, the $N$ mass peak can be clearly observed in the $\mu j$ invariant mass.

The fairly long $N$ proper decay length

$$
\left(c \tau_{N}^{0}\right)^{-1} \simeq \frac{G_{F}^{2} m_{N}^{5}}{16 \pi^{3}}\left(\frac{M_{W}}{M_{W_{R}}}\right)^{4},
$$

characteristic for this portion of parameter space [18], can lead to displaced $N$ decay products. This ranges from sub-millimeter to a few meters, depending on $m_{N}$ and $M_{W_{R}}$ and results in a striking LNV signature with two displaced vertices.

Background estimation. Since lepton number is conserved in the SM, there is no background at parton level for this final state. Nevertheless, there are three distinct ways in which background arises:

1. Electron charge mis-identification and secondary photo-production constitute a background that is hard to understand in absence of real data. Since at this stage one cannot reliably estimate this experimental effect, we study the muon channel free from such issues $[50,51]$.

2. The main prompt muon background comes from pair-production of electroweak gauge bosons, in particular $W Z, Z Z$ and $W^{ \pm} W^{ \pm} j j$, and $t \bar{t}$ production. They also contain non-prompt muons from mesons.

3. Significant background is due to non-prompt muons. This component, likely dominant and not easy to estimate, is due to QCD jets when some hadron is mis-identified as a muon. Although the mis-identification probability is small, the huge QCD production compensates [50, 51]. A realistic estimate will require a knowledge of hadron mis-id within the real detector in the next LHC run. Nevertheless, previous studies indicate this background behaves similarly to the $W Z+Z Z$ background (see supplement of [51]). From that sample, we estimate the QCD mis-id contribution by multiplying the $W Z+Z Z$ background by 2.5 .

Selection criteria and sensitivity. We adopt the default Delphes 3 ATLAS card with muon isolation parameters in agreement with [50] and the anti- $k_{T}$ jet algorithm with $\Delta R=0.4$ and $p_{T j m i n}=20 \mathrm{GeV}$. We demand two same-sign isolated muons and no other leptons, together with $n_{j}$ jets, where $n_{j}=1,2,3$. We require $\mathbb{E}_{T}<30 \mathrm{GeV}$ and leading muon transverse momentum $p_{T}<55 \mathrm{GeV}$. We demand the transverse mass $m_{\mu p_{T}}^{T}<30 \mathrm{GeV}$ and invariant masses $m_{\mu \mu}<80 \mathrm{GeV}, m_{\mu \not p_{T}}<60 \mathrm{GeV}$. The impact of these selection cuts is shown in Tab. I.

For both short and long lived $N$ s the known decay length allows us to impose cuts on the muon vertex transverse displacement $d_{T}$, shown on Fig. 3. We smear the reconstructed vertex with the $p_{T}-\eta$ dependent resolution of $20-40 \mu \mathrm{m}$, as reported in [52]. Since the typical background contains one prompt and one secondary muon, it is effective to cut on both short and long $d_{T}$, on both muons. We employ a sliding window cut, allowing events with $L / 10<d_{T}<5 L$ and optimizing over $L$. This cut is expected to give further control on the multijet QCD background.

\begin{tabular}{|c|c|c|c|c|c|c|}
\hline \hline \multirow{2}{*}{ Process } & \multirow{2}{*}{ No cuts } & \multicolumn{5}{|c|}{ Imposed cuts } \\
\cline { 3 - 7 } & & $\mu^{ \pm} \mu^{ \pm}+n_{j}$ & $\mathbb{E}_{T}$ & $p_{T}$ & $m_{T}$ & $m_{\text {inv }}$ \\
\hline$W Z$ & $2 \mathrm{M}$ & 544 & 143 & 78 & 40 & 20 \\
$Z Z$ & $1 \mathrm{M}$ & 55 & 29 & 16 & 12 & 8 \\
$W^{ \pm} W^{ \pm} 2 j$ & $389^{\dagger}$ & 115 & 16 & 5 & 3 & 1 \\
$t \bar{t}$ & $10 \mathrm{M}^{\dagger}$ & 509 & 97 & 40 & 22 & 14 \\
\hline Signal $(40)$ & 543 & 44 & 43 & 41 & 38 & 37 \\
\hline \hline
\end{tabular}

TABLE I. Number of expected events at the $13 \mathrm{TeV}$ LHC run with $\mathcal{L}=100 \mathrm{fb}^{-1}$ after cuts described in the text. The signal is generated with $40 \mathrm{GeV}, \sin \theta=10 \%, M_{W_{R}}=3 \mathrm{TeV}$ and $n_{j}=1,2,3 .{ }^{\dagger}$ Here we restrict to $t \rightarrow b W$ and $W \rightarrow \mu \nu$.

The final sensitivity is shown in Fig. 4, where a single $N$ with a $100 \% \mathrm{Br}$ to muons is assumed. Given the allowed amount of Higgs mixing $\sin \theta \lesssim 40 \%$, this channel allows to test the LR scale beyond the expected reach $M_{W_{R}} \sim 6 \mathrm{TeV}$ of direct searches [30].

Other models with LNV Higgs decays. The heavy fermions $S$ in standard type-I [7-11] or type-III [58] seesaw couple to the Higgs boson via their Dirac masses $m_{D}$. If these were rather large (unlikely in LRSM [21]) $h \rightarrow \nu S$ decay might be observed [59] and via further mixing $m_{D} / m_{S}$, the LNV channel $h \rightarrow S S$ could open up [32]. Below the $Z$ mass, where such two body Higgs decay is available, constraint on the mixing [51, 60] suppresses it beyond observation.

Further extension with a singlet [61] or spontaneously broken $B-L$ models can generate an observable signal.

Models with supersymmetric R-parity violation, alternative to the seesaw mechanism [62], lead generically to LNV. Same-sign di-lepton decay of the Higgs boson could follow from a fairly large $h \rightarrow \tilde{\chi}^{0} \tilde{\chi}^{0}$ coupling, where $\tilde{\chi}^{0}$ decays to $\ell j j$ thanks to a non-zero $\tilde{l} q q^{\prime}$ term. This scenario may deserve an updated study in light of recent limits. In case of slepton-sneutrino mass degeneracy, the LNV mode appears to be suppressed [63].

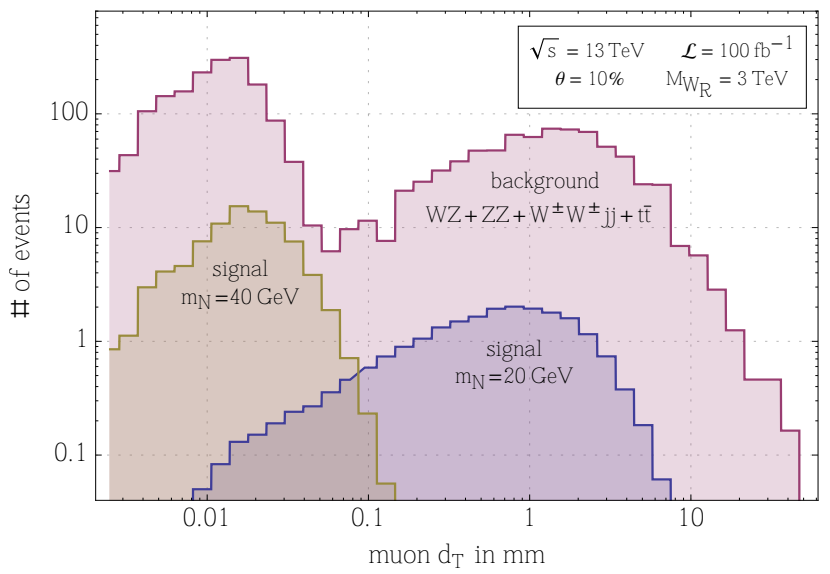

FIG. 3. Reconstructed transverse muon displacement after $\mu^{ \pm} \mu^{ \pm}+n_{j}$ event selection and before other cuts. 


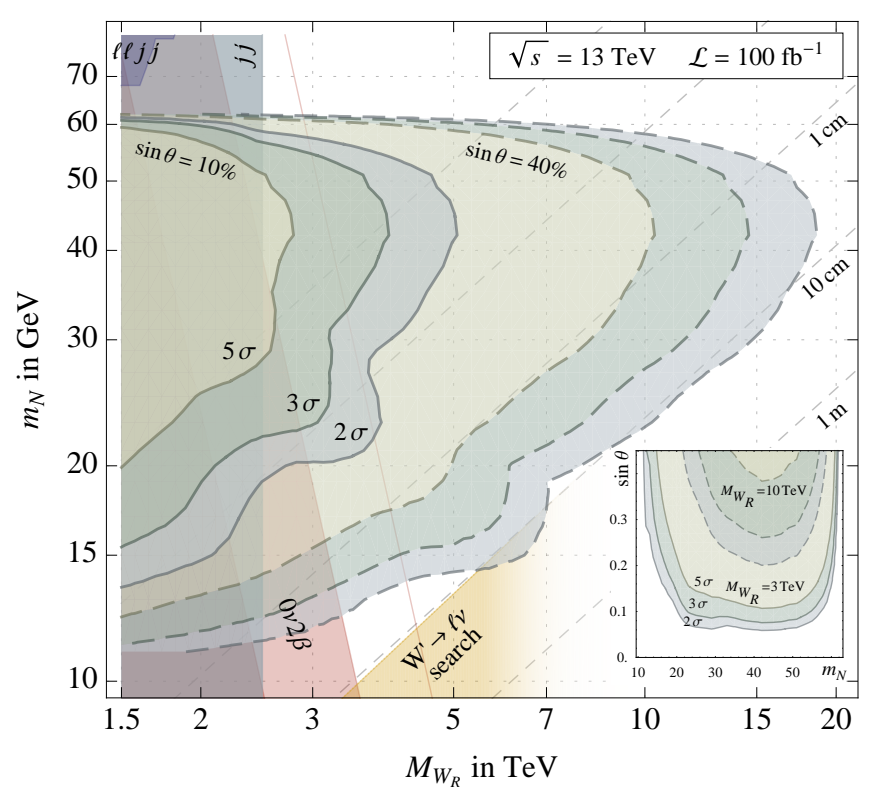

FIG. 4. Expected sensitivity in the $M_{W_{R}}-m_{N}$ plane, in the cases of small and large allowed mixing $\sin \theta$. The current bound from the KS channel [53] is shown in the upper left corner; the limit from di-jet search [54] as the vertical band. The region excluded by the GERDA I [55] $0 \nu 2 \beta$ search is the diagonal shaded region with a factor of 4 uncertainty from matrix elements [56], while the red line marks the sensitivity of GERDA II. The grey dashed lines mark the lab frame decay length of $N$ from Higgs decay and the lower golden triangle the region where the length from $W_{R} \rightarrow \ell N$ decay exceeds $5 \mathrm{~m}$, enabling $W^{\prime} \rightarrow \ell \notin$ searches [57].

Outlook. In the SM, the Higgs mechanism provides masses to charged fermions and leads to a distinct prediction of branching ratios. A completely analogous mechanism operates in the minimal LRSM for $N$ and $W_{R}[7,8]$ where their masses determine the branching ratios of the 'right-handed' Higgs. In this Letter we point out that the mixing between these two bosons leads to $h \rightarrow N N$ and to the interesting possibility of LNV in Higgs decay.

The main results are summarized on Fig. 4. For a large range of allowed $m_{N}$ and $\theta$ values, the $h \rightarrow \ell^{ \pm} \ell^{ \pm}+j e t s$ channel allows to identify the RH neutrino mass peak and can probe the LR scale even beyond the reach of $0 \nu 2 \beta$ or collider searches.

Conceptually, this channel together with evidence of $\theta \neq 0$ can suffice to test the origin of $N$ mass within this scenario. To check Eq. (4) one needs three indepen- dent measurements. The event rate and invariant mass peaks give information on $\theta \times Y_{N}$ and $m_{N}$, respectively. The decay length then constrains $M_{W_{R}}$ via Eq. (6) and finally a global fit on the Higgs data can provide $\sin \theta$. If more than one $N$ were observed, this task simplifies. To complete the understanding of neutrino mass origin, one would clearly like to observe $\Delta$ and the associated gauge boson that provides the gauge symmetry protection. This channel would nevertheless allow to probe the polarization of $N$ decay, similarly to the KS channel [64], even if $W_{R}$ were out of reach. A comprehensive study will be presented in [65].

A number of potential improvements can be identified: i) adding muons with $p_{T}<10 \mathrm{GeV}$ would double the signal; ii) for short lived $N$, tight cuts on displacement could reduce further the QCD multijet background; iii) for boosted long lived $N$ s, the muon tends to merge with the jet and one gets displaced jets. Although a challenge, it may be possible [66] to identify their displacement.

We conclude by pointing out that $e^{+} e^{-}$colliders provide a particularly clean environment for heavy neutrino searches [67]. For the LNV Higgs decay, the relative decrease in production cross-section to $\sigma=0.24 \mathrm{fb}$ at $\sqrt{s} \sim 240 \mathrm{GeV}$ may be compensated by lack of background (only $Z Z$ remains) and large luminosity $\sim 1-$ $10 \mathrm{ab}^{-1}$ [68]. Conversely, a positive signal at LHC without the associated $W_{R}$ discovery would make a case at a high energy hadron collider.

\section{ACKNOWLEDGMENTS}

We thank G. Senjanović for useful discussions on the theoretical part, B. Fuks, O. Mattelaer, M. Selvaggi for correspondence on collider simulation, and F. Giordano, C. Leonidopulos for useful discussions on the CMS and ATLAS experimental features. MN would like to thank B. Bajc, J.F. Kamenik and A. Urbano for useful discussions and E. Nemevšek for help on the manuscript. We would also like to thank G. Senjanović, V. Tello and Y. Zhang for helpful comments on the first version of the manuscript. AM was supported in part by the Spanish Government and ERDF funds from the EU Commission [Grants No. FPA2011-23778, No. CSD2007-00042 (Consolider Project CPAN)] and by Generalitat Valenciana under Grant No. PROMETEOII/2013/007. MN was supported in part by the Slovenian Research Agency. FN was partially supported by the Croatian Science Foundation (HrZZ) project "Physics of Standard Model and Beyond".
[1] P.W. Higgs, Phys. Rev. Lett. 13 (1964) 508.

[2] S. Weinberg, Phys. Rev. Lett. 19 (1967) 1264.

[3] G. Aad et al. [ATLAS Collaboration], Phys. Lett. B 716, 1 (2012) [arXiv:1207.7214 [hep-ex]]; S. Chatrchyan et al. [CMS Collaboration], Phys. Lett. B 716, 30 (2012)
[arXiv:1207.7235 [hep-ex]].

[4] E. Majorana, N. Cim. 14 (1937) 171.

[5] G. Racah, N. Cim. 14 (1937) 322; W.H. Furry, Phys. Rev. 56 (1939) 1184.

[6] G. Feinberg, M. Goldhaber, Proc. Nat. Ac. Sci. USA 45 
(1959) 1301; B. Pontecorvo, Phys. Lett. B26 (1968) 630.

[7] P. Minkowski, Phys. Lett. B 67 (1977) 421.

[8] R.N. Mohapatra, G. Senjanović, Phys. Rev. Lett. 44 (1980) 912.

[9] T. Yanagida, Workshop on unified theories and baryon number in the universe, ed. A. Sawada, A. Sugamoto (KEK, Tsukuba, 1979).

[10] S. Glashow, Quarks and leptons, Cargèse 1979, ed. M. Lévy (Plenum, NY, 1980).

[11] M. Gell-Mann et al., Supergravity Stony Brook workshop, New York, 1979, ed. P. Van Niewenhuizen, D. Freeman (North Holland, Amsterdam, 1980).

[12] J.C. Pati and A. Salam, Phys. Rev. D 10, 275 (1974) [Erratum-ibid. D 11, 703 (1975)]; R.N. Mohapatra and J.C. Pati, Phys. Rev. D 11, 566 (1975); R.N. Mohapatra and J.C. Pati, Phys. Rev. D 11, 2558 (1975).

[13] G. Senjanović and R.N. Mohapatra, Phys. Rev. D 12, 1502 (1975); G. Senjanović, Nucl. Phys. B 153, 334 (1979).

[14] R.N. Mohapatra and G. Senjanović, Phys. Rev. D 23 (1981) 165.

[15] V. Tello, M. Nemevšek, F. Nesti, G. Senjanović and F. Vissani, Phys. Rev. Lett. 106 (2011) 151801 [arXiv:1011.3522 [hep-ph]]; M. Nemevšek, F. Nesti, G. Senjanović and V. Tello, arXiv:1112.3061 [hep-ph].

[16] W.-Y. Keung, G. Senjanović, Phys. Rev. Lett. 50 (1983) 1427.

[17] For a review, see: G. Senjanović, Int. J. Mod. Phys. A 26 (2011) 1469 [arXiv:1012.4104 [hep-ph]]; G. Senjanović, Riv. Nuovo Cim. 34 (2011) 1.

[18] M. Nemevšek, F. Nesti, G. Senjanović and Y. Zhang, Phys. Rev. D 83 (2011) 115014 [arXiv:1103.1627 [hep$\mathrm{ph}]]$.

[19] V. Cirigliano, A. Kurylov, M.J. Ramsey-Musolf and P. Vogel, Phys. Rev. D 70 (2004) 075007 [hep$\mathrm{ph} / 0404233]$.

[20] J.A. Aguilar-Saavedra, F. Deppisch, O. Kittel and J.W.F. Valle, Phys. Rev. D 85 (2012) 091301 [arXiv:1203.5998 [hep-ph]].

[21] M. Nemevšek, G. Senjanović and V. Tello, Phys. Rev. Lett. 110 (2013) 15, 151802 [arXiv:1211.2837 [hep-ph]].

[22] J.F. Nieves, D. Chang and P.B. Pal, Phys. Rev. D 33 (1986) 3324 .

[23] G. Beall, M. Bander and A. Soni, Phys. Rev. Lett. 48 (1982) 848.

[24] R.N. Mohapatra, G. Senjanović and M.D. Tran, Phys. Rev. D 28 (1983) 546; K. Kiers, J. Kolb, J. Lee, A. Soni and G.-H. Wu, Phys. Rev. D 66 (2002) 095002 [hep$\mathrm{ph} / 0205082$.

[25] Y. Zhang, H. An, X. Ji and R.N. Mohapatra, Phys. Rev. D 76 (2007) 091301 [arXiv:0704.1662 [hep-ph]] and Nucl. Phys. B 802 (2008) 247 [arXiv:0712.4218 [hep-ph]].

[26] A. Maiezza, M. Nemevšek, F. Nesti and G. Senjanović, Phys. Rev. D 82, 055022 (2010) [arXiv:1005.5160 [hep$\mathrm{ph}]$.

[27] S. Bertolini, J.O. Eeg, A. Maiezza and F. Nesti, Phys. Rev. D 86 (2012) 095013 [arXiv:1206.0668 [hep-ph]]; S. Bertolini, A. Maiezza and F. Nesti, Phys. Rev. D 88 (2013) 3, 034014 [arXiv:1305.5739 [hep-ph]].

[28] S. Bertolini, A. Maiezza and F. Nesti, Phys. Rev. D 89 (2014) 9, 095028 [arXiv:1403.7112 [hep-ph]];

[29] A. Maiezza and M. Nemevšek, Phys. Rev. D 90 (2014) 9, 095002 [arXiv:1407.3678 [hep-ph]].

[30] A. Ferrari et al. Phys. Rev. D 62 (2000) 013001; S.N. Gni- nenko et al. Phys. Atom. Nucl. 70 (2007) 441.

[31] J.F. Gunion, B. Kayser, R.N. Mohapatra, N.G. Deshpande, J. Grifols, A. Mendez, F.I. Olness and P.B. Pal, PRINT-86-1324 (UC,DAVIS); J. F. Gunion, H. E. Haber, G. L. Kane and S. Dawson, Front. Phys. 80 (2000) 1.

[32] A. Pilaftsis, Z. Phys. C 55 (1992) 275 [hep-ph/9901206].

[33] M.L. Graesser, Phys. Rev. D $\mathbf{7 6}$ (2007) 075006 [arXiv:0704.0438 [hep-ph]]; M.L. Graesser, arXiv:0705.2190 [hep-ph].

[34] https://sites.google.com/site/leftrighthep

[35] A. Falkowski, C. Gross and O. Lebedev, arXiv:1502.01361 [hep-ph]; S.I. Godunov, A.N. Rozanov, M.I. Vysotsky and E.V. Zhemchugov, arXiv:1503.01618 [hep-ph].

[36] D. Buttazzo, F. Sala and A. Tesi, arXiv:1505.05488 [hep$\mathrm{ph}]$.

[37] J. Basecq, J. Liu, J. Milutinović and L. Wolfenstein, Nucl. Phys. B 272 (1986) 145; J. Basecq and D. Wyler, Phys. Rev. D 39 (1989) 870; J.F. Gunion, J. Grifols, A. Mendez, B. Kayser and F.I. Olness, Phys. Rev. D 40 (1989) 1546; N.G. Deshpande, J.F. Gunion, B. Kayser and F.I. Olness, Phys. Rev. D 44 (1991) 837; G. Barenboim, M. Gorbahn, U. Nierste and M. Raidal, Phys. Rev. D 65 (2002) 095003 [hep-ph/0107121].

[38] P. Duka, J. Gluza and M. Zralek, Annals Phys. 280 (2000) 336 [hep-ph/9910279]; K. Kiers, M. Assis and A.A. Petrov, Phys. Rev. D 71 (2005) 115015 [hep$\mathrm{ph} / 0503115]$.

[39] For recent studies of the scalar sector in LRSM, see: G. Bambhaniya, J. Chakrabortty, J. Gluza, M. Kordiaczyńska and R. Szafron, JHEP 1405 (2014) 033 [arXiv:1311.4144 [hep-ph]]; G. Bambhaniya, J. Chakrabortty, J. Gluza, T. Jeliński and M. Kordiaczynska, Phys. Rev. D 90 (2014) 9, 095003 [arXiv:1408.0774 [hep-ph]]; W. Dekens and D. Boer, Nucl. Phys. B 889 (2014) 727 [arXiv:1409.4052 [hep-ph]].

[40] A. Djouadi, Phys. Rept. 457, 1 (2008) [hep-ph/0503172].

[41] K.A. Olive et al. [Particle Data Group Collaboration], Chin. Phys. C 38 (2014) 090001.

[42] G. Senjanović and V. Tello, Phys. Rev. Lett. 114, no. 7, 071801 (2015) [arXiv:1408.3835 [hep-ph]]; G. Senjanović and V. Tello, arXiv:1502.05704 [hep-ph].

[43] A. Alloul, N.D. Christensen, C. Degrande, C. Duhr and B. Fuks, Comput. Phys. Commun. 185 (2014) 2250 [arXiv:1310.1921 [hep-ph]].

[44] A. Roitgrund, G. Eilam and S. Bar-Shalom, arXiv:1401.3345 [hep-ph].

[45] J. Alwall, R. Frederix, S. Frixione, V. Hirschi, F. Maltoni, O. Mattelaer, H.-S. Shao and T. Stelzer et al., JHEP 1407, 079 (2014) [arXiv:1405.0301 [hep-ph]].

[46] T. Sjostrand, S. Mrenna and P.Z. Skands, JHEP 0605 (2006) 026 [hep-ph/0603175].

[47] J. de Favereau et al. [DELPHES 3 Collaboration], JHEP 1402 (2014) 057 [arXiv:1307.6346 [hep-ex]].

[48] E. Conte, B. Fuks and G. Serret, Comput. Phys. Commun. 184 (2013) 222 [arXiv:1206.1599 [hep-ph]].

[49] J.C. Vasquez, "Right-handed lepton mixings at the LHC", [arxiv:1411.5824 [hep-ph]].

[50] G. Aad et al. [ATLAS Collaboration], arXiv:1412.0237 [hep-ex].

[51] V. Khachatryan et al. [CMS Collaboration], arXiv:1501.05566 [hep-ex].

[52] G. Aad et al. [ATLAS Collaboration], Phys. Lett. B 707 (2012) 478 [arXiv:1109.2242 [hep-ex]]; G. Aad et 
al. [ATLAS Collaboration], JINST 7 (2012) P01013 [arXiv:1110.6191 [hep-ex]].

[53] V. Khachatryan et al. [CMS Collaboration], Eur. Phys. J. C 74 (2014) 11, 3149 [arXiv:1407.3683 [hep-ex]].

[54] V. Khachatryan et al. [CMS Collaboration], arXiv:1501.04198 [hep-ex].

[55] M. Agostini et al. [GERDA Collaboration], Phys. Rev. Lett. 111 (2013) 12, 122503 [arXiv:1307.4720 [nucl-ex]].

[56] J. Barea, J. Kotila and F. Iachello, Phys. Rev. Lett. 109 (2012) 042501; J. Barea, J. Kotila and F. Iachello, Phys. Rev. C 87 (2013) 1, 014315 [arXiv:1301.4203 [nucl-th]]; J. Barea, J. Kotila and F. Iachello, Phys. Rev. C 91 (2015) 3, 034304.

[57] V. Khachatryan et al. [CMS Collaboration], arXiv:1408.2745 [hep-ex].

[58] R. Foot, H. Lew, X.G. He and G.C. Joshi, Z. Phys. C 44 (1989) 441; B. Bajc and G. Senjanović, JHEP 0708 (2007) 014 [hep-ph/0612029]; B. Bajc, M. Nemevšek and G. Senjanović, Phys. Rev. D 76 (2007) 055011 [hep$\mathrm{ph} / 0703080]$.

[59] P.S. Bhupal Dev, R. Franceschini and R.N. Mohapatra, Phys. Rev. D 86 (2012) 093010 [arXiv:1207.2756 [hepphl]; C.G. Cely, A. Ibarra, E. Molinaro and S.T. Petcov, Phys. Lett. B 718 (2013) 957 [arXiv:1208.3654 [hep-ph]].
[60] P. Abreu et al. [DELPHI Collaboration], Z. Phys. C 74 (1997) 57 [Erratum-ibid. C 75 (1997) 580].

[61] I.M. Shoemaker, K. Petraki and A. Kusenko, JHEP 1009 (2010) 060 [arXiv:1006.5458 [hep-ph]]; P. Humbert, M. Lindner and J. Smirnov, arXiv:1503.03066 [hep-ph].

[62] Y. Grossman and S. Rakshit, Phys. Rev. D 69, 093002 (2004) [hep-ph/0311310].

[63] T. Banks, L.M. Carpenter and J.F. Fortin, JHEP 0809 (2008) 087 [arXiv:0804.2688 [hep-ph]].

[64] T. Han, I. Lewis, R. Ruiz and Z. g. Si, Phys. Rev. D 87 (2013) 3, 035011 [Phys. Rev. D 87 (2013) 3, 039906] [arXiv:1211.6447 [hep-ph]].

[65] A. Maiezza, M. Nemevšek and F. Nesti, work in progress.

[66] P. Schwaller, D. Stolarski and A. Weiler, [arXiv:1502.05409 [hep-ph]]; S. Bobrovskyi, W. Buchmuller, J. Hajer and J. Schmidt, JHEP 1109 (2011) 119 [arXiv:1107.0926 [hep-ph]].

[67] A. Blondel et al. [FCC-ee study team Collaboration], arXiv:1411.5230 [hep-ex]; A. Das and N. Okada, Phys. Rev. D 88, no. 11, 113001 (2013) [arXiv:1207.3734 [hepph]]; S. Banerjee, P.S.B. Dev, A. Ibarra, T. Mandal and M. Mitra, arXiv:1503.05491 [hep-ph].

[68] M. Bicer et al. [TLEP Design Study Working Group Collaboration], JHEP 1401 (2014) 164 [arXiv:1308.6176 [hep-ex]]. 